\title{
Automated Promotion of Technology Acceptance by Clinicians Using Relational Agents
}

\author{
Timothy Bickmore ${ }^{1}$, Laura Vardoulakis ${ }^{1}$, Brian Jack², and Michael Paasche- \\ Orlow $^{2}$ \\ ${ }^{1}$ College of Computer and Information Science, Northeastern University, Boston, MA, USA \\ \{bickmore,laurap\}@ccs.neu.edu \\ ${ }^{2}$ Boston University School of Medicine, Boston Medical Center, Boston, MA, USA \\ \{brian.jack, michael.paasche-orlow@bmc.org\}@bmc.org
}

\begin{abstract}
Professionals are often resistant to the introduction of technology and can feel threatened if they perceive the technology as replacing some aspect of their jobs. We anticipated some of these problems in the process of introducing a bedside patient education system to a hospital, especially given that the system presents itself as a "virtual discharge nurse" in which an animated nurse agent interacts with patients using simulated face-to-face conversation. To increase acceptance by nursing staff we created a version of the character designed to build trust and rapport through a personalized conversation with them. In a randomized trial, we compared responses after 15 minute in-service briefings on the technology versus responses to the same briefings plus a personalized conversation with the agent. We found that the nurses who participated in briefings that included the personalized conversation had significantly greater acceptance of and lower feelings of being threatened by the agent.
\end{abstract}

Keywords: Relational agent, embodied conversational agent, technology acceptance, medical informatics.

\section{Introduction}

Despite billions in recent US federal spending targeted at clinical information systems, significant barriers to adoption of health information technology (IT) remain [1]. Some of the most significant barriers center on acceptance of the technologies by the clinicians who use them. Although financial incentives may motivate proprietors, it remains to be seen how effective these incentives will be at changing attitudes and actual use behavior by clinicians on the front lines of care who do not stand to personally profit from the incentives, especially if they feel threatened by the technology being introduced.

A significant amount of research has been conducted over the last two decades on the factors that lead to acceptance of a new technology. Attitudes towards a new technology are important even in environments in which use is mandatory, and there are many documented cases of underuse, workarounds, and abandonment of health IT 
[2]. Knowledge of the factors that affect technology acceptance could enable organizations to manipulate these factors to increase acceptance and use of health IT.

The most widely used framework for studying technology acceptance is the Technology Acceptance Model (TAM), which posits that an individual's actual use of a technology can be predicted from their stated intention to use the technology, their attitude towards the technology (overall satisfaction), and perceptions of the technology's ease of use and usefulness [3]. Figure 1 shows the originally hypothesized relationships among these factors. The TAM has received empirical support across a number of industries and technologies, typically accounting for $30 \%$ to $40 \%$ of IT acceptance.

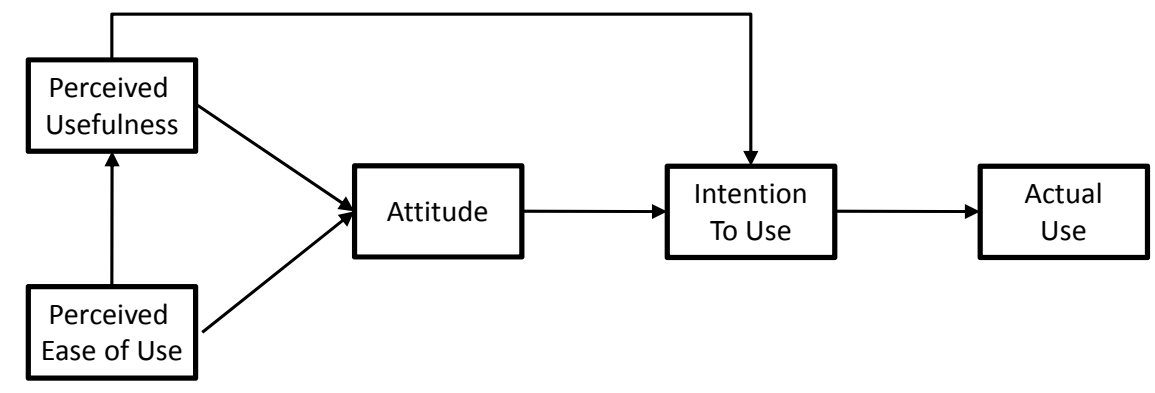

Figure 1. Technology Acceptance Model

Very few studies of the TAM have focused on approaches for promoting acceptance using external influences (such as social influences or persuasive messages), partly due to the wide variety of messages that could be employed, the differential impact these messages have on different user groups, and the lack of temporal models explaining the time course of influences [4]. One framework that has been used widely in studies of attitude change and persuasion is the elaboration likelihood model, in which attitude change is hypothesized to be effected through either "central route" cues (argument quality, appealing to reason) or "peripheral route" cues (meta-information about a message or source, such as the recipient's trust in the source)[5]. This model has been used in studies of persuasion to improve technology acceptance, demonstrating that central route messages can significantly influence perceived usefulness and peripheral route cues can significantly influence both perceived usefulness and attitude towards the technology [4].

In the current study we sought to introduce a new hospital bedside patient education system into the general medical service at Boston Medical Center as part of an intervention to reduce unnecessary re-hospitalizations (Figure 2) [6, 7]. Boston Medical Center is a 547 bed safety net hospital that serves an urban, 84\% minority, traditionally underserved population. Approximately 58\% of the patients hospitalized at Boston Medical Center have inadequate health literacy. Although the new system was designed to offload a routine task from nurses and improve patient care by augmenting patient education regarding post-hospital discharge self-care, we were concerned that the nursing staff may feel threatened by the technology, since it could be seen as a "virtual discharge nurse" intended to replace certain traditional nursing 
duties. In order to increase acceptance of the technology we designed a version of the patient education system especially tailored for the nursing staff that not only explained how the system worked (central route messages), but attempted to directly establish trust and rapport with the nurses and decrease any feelings of being threatened by the technology (peripheral route cues).

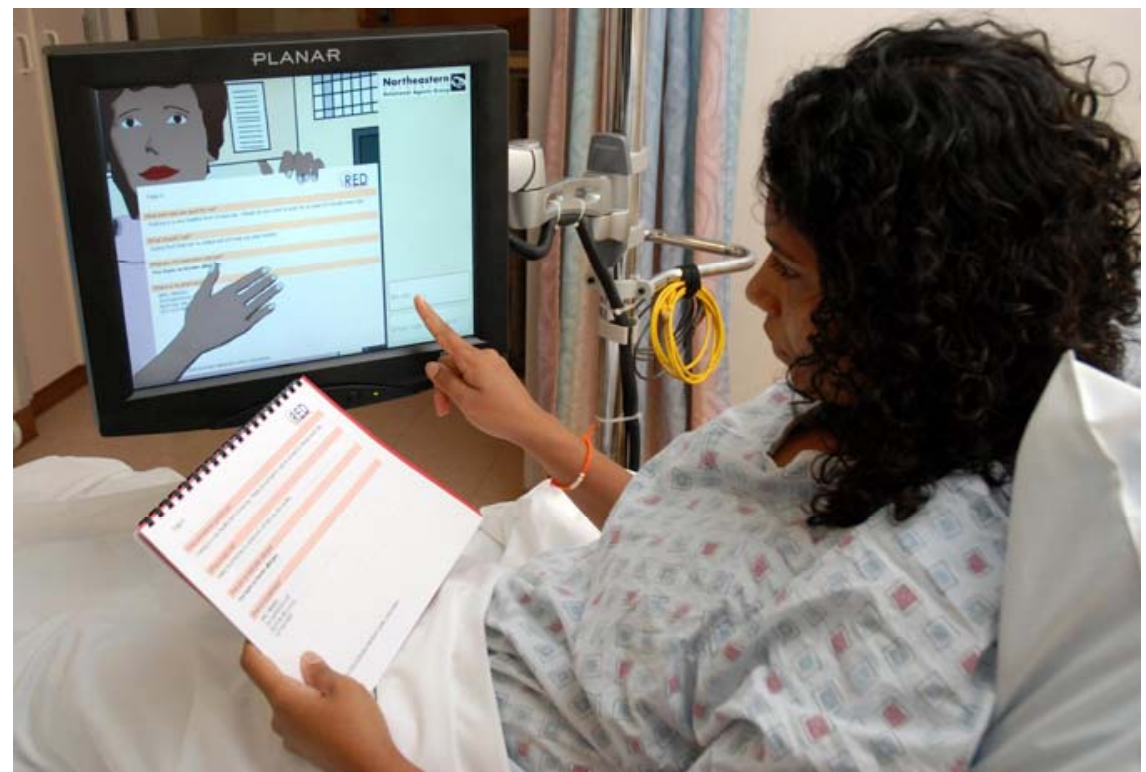

Figure 2. Patient Interacting with the Relational Agent Patient Education System

Intelligent virtual agents that are explicitly designed to build rapport, trust, therapeutic alliance and other forms of working relationships with people have been referred to as "relational agents", and incorporate many of the same behaviors that people use for this purpose, including social chat, expressions of caring and empathy, agreement on values and beliefs, and nonverbal behaviors such as smiling, facial and gestural animation, and close proximity [8]. Relational agents have been used with diverse patient populations for a range of health education and behavior change interventions [6, 9], and are also used in the hospital bedside patient education system (Figure 2). To date, however, relational agents have not been used with professionals for the purpose of increasing technology acceptance in the workplace.

The TAM is appropriate for assessing nurse acceptance even though nurses are not the end users of the system. When the patient education system is deployed in a hospital for routine care, then the nurses on the floor will ultimately determine whether a particular patient gets to use the system or not. We feel this is true in practice, regardless of whether the hospital mandates across-the-board use of the system, or a physician "prescribes" the system for a particular patient. The purpose of the current study is to see what we could do to motivate a nurse to want to wheel the system into the hospital room so their patient could use it. In our pilots and clinical trials we have not had one patient out of over 400 who refused to use this system once 
the study nurse wheeled it bedside and explained how it should be used, so technology acceptance has not been an issue with patients. The TAM Attitude (satisfaction) and Intent to Use measures are very appropriate in this situation with nurses, and the Ease of Use (difficulty for the nurse to set up and difficulty for the patient to use) and Usefulness (both for the patient and in offloading nurse effort) are relevant as well.

In the rest of this paper we describe the design and evaluation of the bedside patient education system, the version we created for the nursing staff, and the results of a pilot evaluation in which we had the nursing staff interact with the tailored system to promote acceptance.

\section{Related Work}

There have been over 20 studies applying the Technology Acceptance Model to clinician acceptance of health IT, demonstrating its applicability in the healthcare context [2]. These studies have involved technologies from electronic medical record systems to telemedicine and PDA-based systems, and investigated acceptance among physicians, nurses and other clinicians. However, we are not aware of any acceptance interventions that used persuasive messages or agents to promote acceptance among target users, including health professionals.

\section{The Virtual Nurse System}

The hospital discharge virtual agent system teaches patients about their post-discharge self-care regimen while they are still in their hospital beds. In order to make the system as acceptable and effective as possible with patients who had a wide range of health and computer literacy, we designed the interface to incorporate an animated virtual nurse who embodies best practices in health communication for patients with inadequate health literacy as well as incorporate the relational behaviors described above [9]. The agent is deployed on a wheeled kiosk with a touch screen display attached to an articulated arm that can be positioned in front of patients while they are in bed (Figure 2). The agent speaks using synthetic speech, driven by a hierarchical transition network-based dialogue engine with template-based text generation, and conversational nonverbal behavior is generated using the BEAT text-to-embodiedspeech translation engine [10]. The agent's repertoire of nonverbal behavior includes a range of hand gestures (beats, emblematics, and deictics [11], including deictics at a document held by the agent), headnods, eyebrow raises, gazing at and away from the user, posture shifts [12], visemes, and facial displays of affect. User contributions to the conversation are made via a multiple-choice menu of utterances dynamically updated at each turn of the conversation [9].

Patients spend approximately half an hour with the RA, reviewing the layout and contents of an "After Hospital Care Plan" booklet that contains their personal medical information designed to assist them in transitioning from the hospital to post-hospital care. The paper booklet is given to patients before their conversation with the agent, 
and the agent reviews a digital version of the booklet in the interface, so that patients can follow along with the system's explanation of their paper booklets.

Pilot evaluations of the agent show that it is well liked and accepted by patients, and $74 \%$ said they prefer receiving their discharge instructions from the agent compared to their human doctors or nurses [6]. We have also found significantly higher levels of satisfaction among patients with inadequate health literacy [13], low computer literacy, and depressive symptoms [14].

We developed a dialogue script to support a personalized one-on-one conversation between a member of the nursing staff and the agent, which was especially tailored to promote acceptance. Figure 3 shows a fragment of a sample interaction that typically lasts 10 minutes. The dialogue was authored to support three major objectives. First, the agent explains how it works, demonstrating how it simulates face-to-face conversation using animated conversational behavior (lines 12-17). Second, the agent explains how it was designed to provide benefits to the patients it interacts with as well as the nurses and the hospital (lines 6-8, 14, 18). Finally, a wide range of agent relational behavior was designed into the conversation to establish trust and rapport with nurses, following work that used this same behavior in automated health counselor agents to establish therapeutic alliance relationships with patients [9]. This included: close forms of greeting and farewell (calling the nurse by his or her given name, lines 1, 20); expressing happiness to see the nurse (line 3) and desire to work together with the nurse (line 8); social chat (lines 10-11); complimenting the nurse (lines 4-5); and desire to talk to the nurse again (lines 20). In addition, the simple act of conducting a sample conversation with the agent provided nurses with first-hand knowledge of how the interaction worked and a sample of the patient experience.

\section{Evaluation}

To evaluate whether one-on-one conversations with the virtual agent promoted acceptance of the system among nurses, we conducted a randomized controlled trial of how the agent was introduced to the nursing staff. All introductions were conducted at regularly scheduled nursing in-service meetings. At these meetings, all nurses received a 15-minute presentation accompanied by PowerPoint slides that described the agent based intervention designed to reduce 30 day all cause rehospitalizations. This presentation included a description of the study purpose and protocol, and a brief description of the agent including a static image of the agent and the interface. Following this presentation, volunteers were recruited for the study, told it would take another 30 minutes of their time, and offered a \$10 gift certificate in compensation for their participation. The study was approved by the Boston Medical Center IRB, and all participants provided informed consent. 
1. A: Hello there! My name is Elizabeth. And you are Mary, is that right?

2. U: Yes, that's right.

3. A: It's great to meet you Mary!

4. A: The researchers and clinicians I have been working with have told me how hard you all work, and you all deserve a pat on the back!

5. U: Thank you!

6. A: I am here at Boston Medical Center to help patients learn about their discharge instructions. I would like to help the hospital become more efficient and modernized, to provide a better environment for staff, patients, and their families.

7. U: Sounds good.

8. A: I am hoping that we can work together and keep BMC one of the greatest hospitals in Boston!

9. U: Great!

10. A: Speaking of Boston, how about those Red Sox?

11. U: They are really something! ...

12. A: So by now, you are probably wondering how I work?

13. U: Yes, actually I was.

14. A: I am glad you want to know about me. Well, first I want to tell you more about who I am. I am a virtual computer agent that was created by computer scientists at Northeastern University. These scientists are interested in using computer characters like me, to help patients in the hospital understand their medications, follow-up appointments, and diagnosis, and feel confident in knowing all of this information when they leave the hospital, in order to help reduce the chance of a re-hospitalization.

15. A: Now that you know how I speak, you will understand how I am able to move. I am also programmed to move or gesture in certain ways, to seem more human like, and to emphasize specific topics. My creators have found this helps with patients' acceptance of me.

16. A: For example, if I am happy, I can smile like this.

17. A: If I am concerned, I will look like this.

18. A: My greatest advantage is that I can explain things to the patient as many times as they like. Since I am a computer, I never get tired or bored. I can also make sure that the patient understands their discharge instructions, by giving them a quiz, on the important aspects of their medications and appointments. If they do not get the answer correct, I can go back over their booklet, and explain it in a different way, to make sure that they understand.

19. U: Okay. ...

20. A: Well, I won't take up anymore of your time today. Thank you for talking with me. It was great to meet you Mary, and I hope we can see each other again sometime. Have a great day and I hope to see you again soon! Bye!

21. U: It was great to meet you too!

Figure 3. Excerpt from Acceptance Promotion Dialogue Lines beginning with "A:” are agent utterances; "U:” are User utterances. 


\subsection{Participants}

Eighteen nurses volunteered to participate in the study. Participants had zero to 30 years of service (average 10.8) and scored at the midpoint on a measure of computer literacy (average 4.2 on a composite scale of $1=$ no experience to $7=$ =xpert) and moderately high on attitude towards computers (average 5.4 on a composite scale of $1=$ negative attitude to $7=$ =positive attitude) [15].

\subsection{Measures}

Primary self-report outcome measures were adapted from prior TAM studies for Perceived Usefulness, Ease of Use, Attitude, and Use Intention [3]. In addition, we used scale self-report items to assess how threatened the nurses were by the agent, and questions about nurses' professional and social relationship with the agent. All items are shown in Table 1.

\subsection{Procedure}

Recruited nurses were randomized into intervention and control groups. Intervention participants were taken to another room where they each conducted a personalized acceptance promotion conversation with the virtual agent as described above. Prior to each conversation, a research assistant entered the participant's given name into the system so that the agent could address the participant by name. agent conversations were conducted on the same mobile touch screen computer cart used for hospital bedside patient education (Figure 2). Immediately following this conversation, intervention participants were asked to fill out the self-report questionnaire for all measures.

In contrast, control group participants were asked to fill out the same questionnaire immediately following recruitment (i.e., shortly after the in-service presentation).

Approximately 30 days after the in-service briefing an attempt was made to reach all study participants and have them fill out the outcome measures portion of the questionnaire again.

\subsection{Results}

Exactly half of the 18 participants were randomized into each group. There were no significant differences between intervention and control groups on years of service, computer literacy or attitudes towards computers. 
Table 1. Self-Report Measures and Outcomes

Significance values from t-tests for independent means.

"Elizabeth" is the name of the Virtual Agent

After In-Service

$(\mathrm{N}=18)$

\section{Questions}

Perceived Usefulness $(\alpha=.95)$

(1=disagree completely ... 7=agree completely)

Agent Control p

6.4

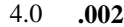

\begin{tabular}{ccc} 
Agent & Control & p \\
\hline 5.5 & 3.8 & .1
\end{tabular}

(1) Using the Elizabeth patient education system enhances my effectiveness on the job. (2) Using the Elizabeth patient education system allows me to get my work done more quickly. (3) The Elizabeth patient education system is useful to me.

Ease of Use $(\alpha=.90)$

(1=disagree completely ... 7=agree completely)

(1) The Elizabeth patient education system is easy to use. (2) The Elizabeth patient education system is easy to operate. (3) It is easy to get the Elizabeth patient education system to do what I want it to do.

Attitude

How satisfied are you with the Elizabeth patient education system?

Use Intention $(\alpha=.94)$

(1=disagree completely ... 7=agree completely)

(1) If I could, I would use the Elizabeth patient education system as a routine part of my job over the next year. (2) If I could, I would use the Elizabeth patient education system at every opportunity over the next year. (3) I would recommend the Elizabeth patient education system to my patients.

I feel threatened by Elizabeth.

(1=disagree completely ... 7=agree completely)

I consider Elizabeth part of my healthcare team.

(1=disagree completely ... 7=agree completely)

How would characterize your relationship with

Elizabeth?

(1=complete stranger ... 7=close friend)

How much do you trust Elizabeth?

(1=not at all ... 7=very much)

$\begin{array}{llllll}1.4 & 3.4 & . \mathbf{0 2 5} & 2.0 & 3.5 & .308 \\ 6.6 & 3.5 & \mathbf{. 0 0 2} & 6.8 & 3.3 & \mathbf{. 0 0 1} \\ 5.0 & 3.5 & .144 & 4.8 & 2.7 & .059 \\ & & & & & \\ 6.0 & 4.4 & . \mathbf{0 4 2} & 6.0 & 4.0 & .078\end{array}$


Outcome results are presented in Table 1. Intervention group participants scored the RA significantly higher on Perceived Usefulness, Ease of Use, and Attitude (overall satisfaction), and expressed a significantly greater intention to use the agent system, compared to those in the control group. Intervention group participants also expressed a significantly higher degree of trust in the agent character, felt that she was more a part of their "healthcare team", and felt significantly less threatened by the agent character, compared to those in the control group.

At 30 days, we were only successful in obtaining follow-up measures from eight participants. However, the trends on all measures remained consistent, and several differences between the groups remained statistically significant, including Ease of Use and Use Intention.

\section{Conclusions and Future Work}

The rapport and trust-building dialogue was successful in increasing trust in the virtual agent, and significantly increased nurses' intention to use the patient education system. Whether this effect was due to increases in Perceived Usefulness or Ease of Use (the primary determining factors according to the Technology Acceptance Model) cannot be determined, since they were both significantly higher in the intervention group. We also cannot determine whether the influence was achieved via central or peripheral route persuasion cues (per the elaboration likelihood model), since these were not manipulated separately: future studies that parametrically vary these factors are needed. However, the combination of a personalized conversation with the RA and the specific promotion dialogue together did lead to a significant reduction in nurses' feelings of being threatened by the virtual agent.

This study has several limitations, including the small sample size and lack of an actual behavioral measure of system use following the intervention. In our case we could not measure this since the decision to use the agent with a patient was always made by research staff and the nurses in this technology acceptance study never had any actual choice or say in the decision. However, previous TAM studies have demonstrated that self-reported Use Intention (as measured here) is a highly significant predictor of actual use of voluntary use systems. The sample size was also too small to verify the causal path relationships among the TAM factors. This was also one of the primary reasons why we chose to use the original, simpler TAM and focus on the essential elements of attitude and intent to use, rather than using one of the more recent and complex models (e.g. UTAUT [16]). In addition, some of the differences observed may have been simply due to the intervention group having direct experience with the system being evaluated whereas the control group only had indirect experience, although neither group experienced the hospital discharge interaction that was given to patients. We also did not control for the additional time and attention given to the intervention group nurses. Finally, our approach, as with any attempt at manipulating acceptance, will likely have little impact if the IT system at issue is inherently either extremely acceptable or unacceptable.

Relational behaviors have been successfully used in animated conversational agents to build therapeutic alliance relationships with patients to increase satisfaction, 
engagement and adherence to medication and other health behavior regimens [9]. However, to our knowledge, this is the first time that automated relationship-building behavior by an interface agent has been used with clinicians, and the first time it has been successfully used to promote technology acceptance.

Future work includes assessment of whether trust-building conversations such as the one presented here lead to actual increased use, whether ongoing relational agent conversations are effective at maintaining clinician acceptance over time, and whether this technology can be used to influence other aspects of clinician behavior. The techniques described here could also be used in many other areas within and beyond healthcare to promote technology acceptance among professionals.

Acknowledgments. Thanks to Julie O’Donnell, Shaula Forsythe, and Gail Burniske for their assistance with the study, and to the many members of the Re-Engineered Discharge (RED) project team at Boston Medical Center and Northeastern University. This work was funded by grant R01 HL081307 from the US NIH National Heart, Lung, and Blood Institute.

\section{References}

1. Blumenthal, D.: Stimulating the Adoption of Health Information Technology. The New England Journal of Medicine 360, 1477-1479 (2009)

2. Holden, R., Karsh, B.: The technology acceptance model: its past and its future in health care. Journal of Biomedical Informatics (2009)

3. Davis, F.D.: User acceptance of information technology: System characteristics, user perceptions and behavioral impacts. International Journal of Man-Machine Studies 38, 475487 (1993)

4. Bhattacherjee, A., Sanford, C.: Influence Processes for Information Technology Acceptance: An Elaboration Likelihood Model. MIS Quarterly 30, 805-825 (2006)

5. Petty, R., Cacioppo, J.: Attitudes and Persuasion: Classic and Contemporary Approaches. Westview Press, Boulder, CO (1996)

6. Bickmore, T., Pfeifer, L., Jack, B.W.: Taking the Time to Care: Empowering Low Health Literacy Hospital Patients with Virtual Nurse Agents. ACM SIGCHI Conference on Human Factors in Computing Systems (CHI) (2009)

7. Bickmore, T., Pfeifer, L., and Paasche-Orlow, M.: Health Document Explanation by Virtual Agents. Intelligent Virtual Agents, pp. 183-196, Paris (2007)

8. Bickmore, T., Picard, R.: Establishing and Maintaining Long-Term Human-Computer Relationships. ACM Transactions on Computer Human Interaction 12, 293-327 (2005)

9. Bickmore, T., Gruber, A., Picard, R.: Establishing the computer-patient working alliance in automated health behavior change interventions. Patient Educ Couns 59, 21-30 (2005)

10. Cassell, J., Vilhjálmsson, H., Bickmore, T.: BEAT: The Behavior Expression Animation Toolkit. SIGGRAPH '01, Los Angeles, CA, pp. 477-486. (2001)

11. McNeill, D.: Hand and Mind: What Gestures Reveal about Thought. Cambridge University Press, Cambridge (1992)

12. Cassell, J., Nakano, Y., Bickmore, T., Sidner, C., Rich, C.: Non-Verbal Cues for Discourse Structure. Association for Computational Linguistics, Toulouse, France, pp. 106-115. (2001) 
13. Bickmore, T., Pfeifer, L., Byron, D., Forsythe, S., Henault, L., Jack, B., Silliman, R., Paasche-Orlow, M.: Usability of Conversational Agents by Patients with Inadequate Health Literacy: Evidence from Two Clinical Trials. Journal of Health Communication 15, 197210 (2010)

14. Bickmore, T., Mitchell, S., Jack, B., Paasche-Orlow, M., Pfeifer, L., ODonnell, J.: Response to a Relational Agent by Hospital Patients with Depressive Symptoms. Interacting with Computers 22, 289-298 (2010)

15. Nickell, G.S., Pinto, J.N.: The computer attitude scale. . Computers in Human Behavior 2, 301-306 (1986)

16. Venkatesh, V., Morris, M., Davis, G., Davis, F.: User acceptance of information technology: toward a unified view. MIS Quarterly 27, 425-478 (2003) 